\title{
A method to improve integrated product service offerings based on life cycle costing
}

\author{
Tomohiko Sakao and Mattias Lindahl
}

\section{Linköping University Post Print}

\section{Tweet}

N.B.: When citing this work, cite the original article.

Original Publication:

Tomohiko Sakao and Mattias Lindahl, A method to improve integrated product service offerings based on life cycle costing, 2015, CIRP annals.

http://dx.doi.org/10.1016/j.cirp.2015.04.052

Copyright: Elsevier

http://www.elsevier.com/

Postprint available at: Linköping University Electronic Press

http://urn.kb.se/resolve?urn=urn:nbn:se:liu:diva-117510 


\title{
A method to improve integrated product service offerings based on life cycle costing
}

\author{
Tomohiko Sakao, Mattias Lindahl \\ Department of Management and Engineering, Linköping University, Sweden \\ Submitted by J.-G.Persson (1), Stockholm, Sweden
}

Although a few papers have reported on life cycle cost (LCC) analysis of integrated product service offerings (IPSOs), insight on how to improve IPSOs based on LCC analysis is missing. This paper presents a method and an Excel and MATLAB-based tool that support IPSO design by employing LCC analysis, both from the provider and customer perspectives. This method takes advantage of exchangeability between products and services, being enabled within IPSO design. The method has been applied to an existing IPSO and potential improvements have been identified, e.g. one cheap component causing high LCC that could be reduced significantly by redesign.

Lifecycle; Cost; Service

\section{Introduction}

Manufacturers in developed countries face increasing demand from the markets to decrease costs, and from society to decrease environmental impacts. In responding to these demands, the lifecycle perspective is essential and there are different lifecyclebased concepts and methodologies to support manufacturers' designs. Among them are integrated product service offerings (IPSOs) [1], which can be defined as offerings combining physical products and services that have been integrated and optimized from a lifecycle perspective in relation to customer value (modified from Meier et al. [2]). Note that IPSO has much in common with Industrial Product-Service System (IPS2) [2], but this article uses IPSO to emphasize the importance of their integration. Another is life cycle cost (LCC) analysis, a method for evaluating solutions.

A main objective of LCC analysis is to quantify the total cost, i.e. financial costs associated with an offering throughout its full lifecycle [3,4]. Examples of costs are from research and development, sales, design, construction, operation, maintenance, and replacement or disposal [3,5]. Unlike Life Cycle Assessment (LCA) [6], there is no overall, general ISO standard for LCC, but standards have been developed e.g. for the building and constructed asset sectors [7]. The IEC, however, has developed one standard that provides a general introduction to the concept and covers all applications [5]. Their standard is intended for general application by both purchasers and providers.

Although LCC analysis is argued to be useful for both economic and environmental aspects [8], it has not proliferated in the literature as compared to LCA according to Umeda et al. [9]. When it comes to LCC of IPSOs, development of scientific knowledge is even more limited. IPSOs are often acknowledged by companies to contribute to a reduced cost [10], but it is not clear how much can be earned quantitatively by IPSOs in economic terms. Only a few papers are found in the literature on the subject, e.g. Smith [11], who reports reduced costs for the buyers of a Rolls-Royce offering called "Power by the Hour" in aggregation per year, i.e. not per offering. A recent paper shows reduced costs for providers in reference to products sold without describing the details of the adopted procedure for LCC [1].

From a methodological viewpoint, Settanni et al. [12] discuss the cost object, scope, and how to compute for LCC of IPSOs. A model to calculate LCC for oilfield service providers has been developed and implemented as spreadsheet-based software by Marten and Gatzen [13]. However, none of these articles touch upon how to improve IPSOs based on LCC. There is a need to develop insights for this, as improving an offering from the cost perspective is in general one of the most frequent and important tasks in engineering design.

Therefore, the main goal of the article is to propose a method to support improvement of offerings by employing LCC. In addition, an Excel and MATLAB-based tool is proposed to efficiently apply this method. The method allows a user to adopt perspectives both from provider and customer as well as to compare IPSOs with traditional product sales. The unique feature of the method in the LCC context is its capability to take advantage of interdependency between product and service in IPSO design originating of their integration. To validate the method and tool, they have been applied to an existing IPSO and product sales by a provider. As a result, the offerings as well as different activities and components were evaluated and useful information could be obtained in order to improve the offerings.

Another goal is to report LCC analysis of an IPSO on the market and to discuss general insights about LCC of IPSOs.

The remainder of this article is as follows. Section 2 proposes the method and the tool. Next, Section 3 uncovers results obtained from applying the method and tool to offerings on the market by a provider. Section 4 then discusses the results, while Section 5 concludes the article with a description of future work.

\section{The method and tool for LCC analysis of IPSO}

\subsection{Overview of the method}

The proposed method builds upon several standards and handbooks for LCC (e.g. $[3,5])$, and is also further developed by the authors, who were inspired by LCA [6]. It consists of eight steps in Figure 1. What is unique with this method as compared to general LCC are Steps 4 and 8 . Since those are specific to this 
method, they are further explained in Sections 2.2 and 2.3, respectively, and highlighted in bold font in Figure 1. In order to get a more complete foundation for Step 8, to calculate LCC from both the customer and provider perspective is recommended.

The outcomes are: a) Ranking of activities and components' contribution to an offering's LCC; b) Ranking of offerings; c) Ranking of factors influencing sensitivity; and d) Ranking of IPSO improvement efficiency. The first three outcomes are not unique, while the last is unique with the proposed method.

\begin{tabular}{|l|}
\hline 1. Definition of scope of analysis \\
\hline 2. Definition of system boundaries and data quality \\
\hline 3. Definition of potential offerings \\
\hline 6. Development of function structure \\
\hline 7. Sensitivity analysis \\
\hline \begin{tabular}{l} 
8. IPSO improvement analysis \\
\hline
\end{tabular}
\end{tabular}

Figure 1. Flowchart of the proposed method.

\subsection{Step 4 - Development of function structures}

Based on a modelling technique by Arai and Shimomura [14], the functions for offerings and the means for those functions are described in a tree structure. Each function is deployed into a physical component or a service activity. It is important to describe all the components and activities realizing a function in the function structure with exchangeability. The exchangeability means the feature of being able to exchange efforts for service and physical-product design to improve the overall IPSO characteristics of interdependent product and service. E.g., durability of a part and frequency of maintenance are characteristics of means for a function "maintain the part's condition". In this case, the part and the maintenance are interdependent and these characteristics have exchangeability.

\subsection{Step 8 -IPSO improvement analysis}

This step is based on Step 4 and Outcomes a)-c). The first task, in case multiple offerings are compared, is to identify the most promising offerings that are further improved. Outcome b) is useful for doing this and for each selected offering the next task is to identify what activities and components mainly contribute to the total cost based on Outcome a). In order to prevent potential LCC sub-optimizations (e.g. a change may cause a pro for the customer but a con for the provider), this ought to be done from both the provider and customer perspective.

The most significant cost drivers (functions) are selected, and based on the function structure; alternative solutions for those functions are developed by taking advantage of the exchangeability between interdependent product and service in IPSO design. To do so, Step 8 identifies any set of components and activities realizing a function and calculates the impact on the total LCC by a change of characteristics of the components and activities. The outcome is d) ranking of IPSO improvement efficiency to total LCC. For clarification, see Table 1 for comparison of sensitivity analysis and IPSO improvement analysis. The former examines a change of one parameter's value at a time, while the latter addresses two or more parameters - a set of interdependent component and activity. Both, however, calculate the impact on LCC. This task is an interactive process and ends when the IPSO developers are satisfied with the achieved LCC performance of the modified IPSO (ideally both from the provider and the customer perspective).

Table 1

Comparison of sensitivity analysis and IPSO improvement analysis.

\begin{tabular}{l|l|l} 
& Input & Output \\
\hline Sensitivity analysis & $\begin{array}{l}\text { Uncertainty of component or } \\
\text { Uncertainty of activity }\end{array}$ & $\begin{array}{l}\text { Impact } \\
\text { on LCC }\end{array}$ \\
$\begin{array}{l}\text { IPSO improvement } \\
\text { analysis }\end{array}$ & $\begin{array}{l}\text { Uncertainty of a set of component and } \\
\text { activity with exchangeability }\end{array}$ & $\begin{array}{l}\text { Impact } \\
\text { on LCC }\end{array}$ \\
\hline
\end{tabular}

\subsection{Overview of the tool and its functions}

The tool mainly supports Steps 5-8 of the method and is based on an Excel database in combination with a MATLAB script, as shown in Figure 2. The choice of the Excel format for the database was to enable quick and effective input of data, while the choice of MATLAB for calculations was made to secure fast and easy program calculations. The components are Excel sheets, one for each offering (existing or newly developed ones) and with all data stored, MATLAB scripts for handling calculations and Excel sheets for storing the raw result of the calculations. The MATLAB script also creates a number of graphs for easy overview.

Step 5's data collection is supported by the tool's predefined and still flexible syntax and structure for data collection and structuring, e.g. category, unit, type, cost, start and end and comments. Each stage in the lifecycle is represented by an Excel sheet, and there are also sheets for global data (for all stages) and general information.

Steps 6-8 are supported by the tool's programmed calculations, done with MATLAB and supporting multiple parallel potential offering calculations simultaneously, as seen in Figure 3. Steps 7 and 8 are supported by running the tool's calculator script multiple times, but with sensitivity or IPSO improvement analysis in focus, e.g. by alternating various parameters in the sensitivity analysis. The IPSO improvement analysis is performed in a similar way, i.e. by changing characteristics of key components and activities, and thereafter calculating the impact on the total LCC as well as e.g. changes in ranking between different offerings.

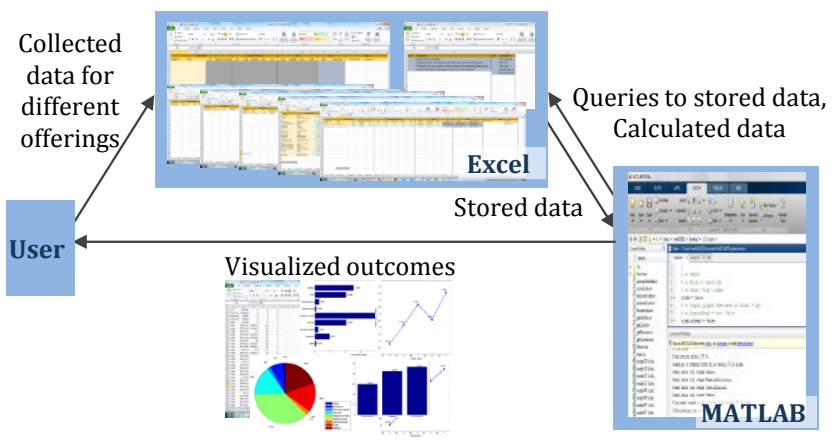

Figure 2. Structure of the tool and exchanged information.

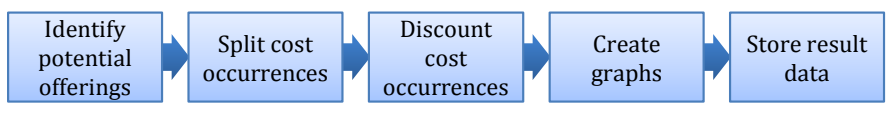

Figure 3. Calculation procedure of MATLAB in the tool.

\section{Empirical findings from a real case at a provider}

\subsection{Circumstances of the provider and their offerings}

This company is an original equipment provider of forklift trucks as well as a direct provider of various kinds of services such as inspection, repair and take-back, to forklift truck users. Their forms of contracts are categorized into two types. One is 
traditional product sales (product, hereafter), where the users can purchase additional services. The lifetime for depreciation is often counted as six years. The other is a kind of IPSO (IPSO, hereafter), where products and services are included in a packaged offering without the product ownership transferred to the users. One of the periods for the company's IPSO contracts is six years, and they have provided IPSOs for many years. They have also launched a monitoring device, which is attached to their product, to record different types of data from products in use.

It should be emphasized that this company has strategically shifted to the role of IPSO provider. Their motivations for this include efforts to provide their customers with higher value and utilization of the remaining value in the products after "end of life". The products have a certain level of complexity, and they found potential to improve their offerings in terms of LCC.

\subsection{Data collection and overview of the empirical research}

The test of the proposed method and tool and the LCC analysis of offerings were done in the fall of 2014, in close collaboration with company staff.

Steps 1-4 were performed in a 1.5 hour workshop with a total of 3 people. It was decided that the scope was to, both from a provider and customer perspectives, compare two different forms of contracts, that is product and IPSO, and to focus on one of their existing customers with a current IPSO contract. The customer has 3 sites with $196(167+18+11)$ warehouse forklift trucks. All sites are located relatively close to each other. The current IPSO contract is for 6 years. From the customer's perspective, all offerings have three phases: purchase, usage and disposal (from the provider's perspective the same offerings include more phases, e.g. production and installation). The function structures for IPSO and Product were developed via discussion between the authors and company staff, both from the provider and customer perspectives. They differ mainly during the usage phase. E.g. in the IPSO structure, the provider is more active in the service activities. The IPSO structure is also more complex when it comes to functions needed.

Step 5 was done by the company staff using a questionnaire based on the offerings' function structures and the method and tool's predefined structure for data collection and structuring. In order to provide data, they used existing data from the specific customer and their overall customer database. Mainly in order to confirm or complement missing data, semi-structured interviews and a second workshop were held by the staff and the authors.

Regarding the data collection, strong trust between the authors and the firm enabled access even to highly business-sensitive data. In addition, calculations regarding the IPSO were always carried out so as to underestimate, rather than overestimate, the IPSO benefits. This means that IPSO benefits are more likely larger in reality than the results presented in Section 3.3.

Steps 6-8 were for practical reasons performed by the authors using the tool. However, it should be noted that these steps could have been done by the company staff, and during the second workshop mentioned above, they actually tested and used the tool for testing different factors' influence on sensitivity. Generated outcomes were discussed during this workshop, and afterwards some modification of ingoing data was done and new calculations were made (following iteration back to Step 5 in Figure 1) and verified by the company staff. In Step 8, most physical components were combined with service activities to realize a function in the described function structures.

\subsection{Outcomes of the proposed method and tool}

Due to the space limitation, the provider's perspective is only partially presented, and the focus is on the customer's perspective. These data have been carefully managed and agglomerated so that the final results do not reveal sensitive secrets, yet maintain the sufficient sources for scientific argumentation.

Outcome a) is described as follows in the case of product sales only, due to the space limitation. The dominant cost in the purchase is the forklift truck. Within the usage, except for the cost of truck drivers, different service activities and spare parts accounted for approximately $60 \%$ and $40 \%$, respectively. The service activities included repair as the major contributor.

Figure 4 shows Outcome b) from the customer's perspective. As shown, the IPSO is in total less costly than the product. The predominant cost in the product purchase phase is the one for forklift trucks, and the use phase consists of various types of costs, e.g. for service and spare parts, that occur over the 6-year life phase. In the IPSO case, the cost for ingoing forklift trucks are allocated over the offering's 6-year lifecycle as well as all other costs, e.g. service and spare parts.

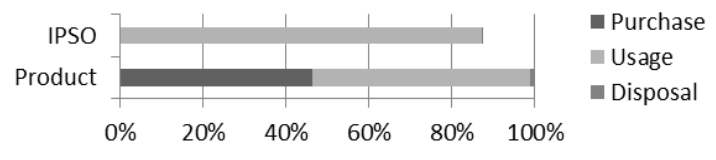

Figure 4. Comparison of IPSO and product in total LCC from the purchaser's perspective and lifecycle phases' contribution to the LCC.

From the provider's perspective, the IPSO seems to be preferable. The reason is that the provider will be involved in more functions when providing an IPSO than applying conventional product sales. This implies for the IPSO an increased number of points of contacts with the customer and at the same time increased potential to further optimize the total LCC.

Regarding Outcome c), the frequency of service activities is found to be an important sensitivity factor. These occur many times over many years and if ingoing data is incorrect or imprecise, the accumulated result will have a significant effect on the total result.

Concerning Outcome d), as an example, dependencies of tires on other activities and components were investigated based on Outcomes a) and c) and "provide repair of tires" was identified as an important function. Thus, both decreasing and increasing the cost for a tire and its related components, and redesign of the wheel suspension, in combination with the affected direct (for repair) and indirect (for standstills) cost for repair, was investigated. The result was that increased durability, in combination with a redesign of the wheel suspension (and thereby increased cost of the tire related components), is one of the most efficient ways to reduce total LCC. This is valid for both product and IPSO.

In addition, dependencies of repair were investigated and the monitoring device was identified through a function called "provide qualified conditions for forklift trucks". Since the labour cost for the forklift truck driver is of major importance, reducing the time for repair is very interesting from a customer perspective. Building more automatic monitoring into the device was also viewed as an efficient option. This option would increase the forklift's cost, but the decrease in the cost of repair (e.g. prevention of unwanted standstills) would outweigh it. Further, this was confirmed positive also via decrease of another cost, i.e. decrease of labour cost for the forklift truck driver by increase of the accessibility (availability) of the forklift truck.

\subsection{Feedback from staff at the provider}

The outcomes of the proposed method and tool have been considered useful and interesting with regard to design of the forklift and related services. Furthermore, in line with the authors' intention, they consider that the proposed method and 
tool provide an effective, structured, fast and easy way to provide reliable, repetitive and pedagogic results regarding e.g. ranking of factors influencing sensitivity and IPSO improvement efficiency. Currently they do not, despite the company's long history of providing IPSOs, have any method or tool that can provide the support as the proposed method and tool can. Finally, the company staff have identified and provided several useful practical applications of the method and tool that are worth further consideration and investigation. These are utilized for generalized discussion in Section 4.3.

\section{Discussion}

\subsection{Effectiveness of the proposed method and tool}

Section 3 demonstrated that the proposed method and tool are effective in a provider's real practice to support evaluation of potential IPSOs and identification of efficient improvement options. The information required for the method can be obtained from IPSO providers in general and at an early stage of improvement. Thus, the method and tool have wide applicability to IPSOs and create a high impact on design efficiency due to their use in an early stage.

It should be noted that the proposed IPSO improvement analysis gives additional improvement opportunities compared to addressing either product or service improvement. In addition, a current composition of LCC does not hinder successful application of the analysis. E.g. in case a product cost is dominant in an LCC, the analysis could suggest an improvement option to increase maintenance cost and make the product cheaper - this cannot be derived if addressing products and services separately.

The additional efforts as compared to ordinary LCC are Steps 4 and 8. Step 4, i.e. development of function structure, creates fundamental information about an offering and is useful for many other purposes in lifecycle engineering. Partly because of this, the additional efforts could be outweighed by the additional valuable informationfrom the method including Outcome d).

Developed function structures may have variety between method users. This variety, however, is not unique to this context and experienced designers should be able to collect sets of components and activities realizing a function based on the experience of the case study. Therefore, the method and the tool can be regarded as robust in this sense.

\subsection{Results from the LCC analysis}

In this case, the IPSO was found to be better than the product from the customer's perspective. One reason identified, in line with the basic idea of IPSO, is an integrated offering, which is optimized in order to avoid potential sub-optimization and to utilize ingoing components in a more efficient and effective way, e.g. by enabling the provider to take back forklift trucks for refurbishment and reuse in other offerings. Scale of economy is also a key factor; the provider can build up both knowledge and split cost within the IPSO in a way a normal customer cannot in the product purchase. The result, therefore, is unsurprising and in line with other authors' conclusions (e.g. [11]).

The LCC from the provider's perspective was not extensively shown in this article, but comparison with the other cases (e.g. [1]) will be a focus of future work by the authors.

\subsection{Scientific contribution}

The major scientific novelty of this article exists in the validated additional steps and outcome as compared to ordinary LCC analysis. In particular, the use of exchangeability in IPSO design is new and also impossible if the design parameters are concerned with either a physical product or service activities. I.e., this opportunity has been brought about by IPSO design. This enables the choice of a cheaper option to create value regardless of the target to be improved, be it product or service.

\subsection{Practical contribution}

Practical contributions of the method and tool are summarized as follows. First, the tool contributes to decrease the time and risk of human error in LCC analysis through its automated calculation procedure. In practice, it is virtually impossible within design activities to run the calculation and react to the results without such a tool. Second, the method and the tool are not expensive to learn and implement at a company. Third, the tool can be used to discuss the impact of a certain alternative on e.g. profits, either within the provider (e.g. with the marketing and sales staff) or with its customer/supplier. Fourth, they are applicable to a potential customer as well as an existing one.

\section{Conclusion and future work}

This article proposes a method and tool to evaluate and improve IPSOs by employing LCC analysis. In addition, the article reports on an LCC analysis of an IPSO and product sales on the market with some general insights about LCC of IPSOs.

Future work will include: 1 . investigation of the LCC analysis process such as data allocation specific to the context of IPSOs with referring to e.g. [12], 2. handling more complex cases such as analysis of a customer's multiple contracts, and 3. analysing LCC from the provider's perspective as stated in Section 4.2.

\section{Acknowledgements}

The authors would like to express special gratitude to the anonymous company for their finance and active cooperation in the empirical research. Our gratitude is extended to Emanuel Reneby from Linköping University for coding the tool and support with carrying out Steps 6 and 7 on the case.

\section{References}

[1] Lindahl, M., Sundin, E., Sakao, T., 2014, Environmental and Economic Benefits of Integrated Product Service Offerings Quantified with Real Business Cases, Journal of Cleaner Production, 64: 288-296.

[2] Meier, H., Roy, R., Seliger, G., 2010, Industrial Product-Service Systems - IPS ${ }^{2}$, Annals of the CIRP, 59/2: 607-627.

[3] Swarr, T.E., Hunkeler, D., Klopffer, W., Pesonen, H.-L., Ciroth, A., Brent, A.C., Pagan, R., 2011, Environmental Life Cycle Costing: A Code of Practice, SETAC.

[4] Rebitzer, G., Hunkeler, D., 2003, Life cycle costing in LCM: ambitions, opportunities, and limitations, The International Journal of Life Cycle Assessment, 8/5: 253-256.

[5] IEC, 2004, Dependability management - Part 3-3: Application guide - Life cycle costing, IEC 60300-3-3, The International Electrotechnical Commission.

[6] ISO, 1997, ISO 14040 -Environmental management - Life cycle assessment Principles and framework-, International Organization for Standardization.

[7] ISO, 2000, Building and Constructed Assets - Service Life Planning: Part 1 General Principles, ISO 15686-1, International Organization for Standardization.

[8] Gluch, P., Baumann, H., 2004, The life cycle costing (LCC) approach: a conceptual discussion of its usefulness for environmental decision-making, Building and Environment, 39/5: 571-580.

[9] Umeda, Y., Takata, S., Kimura, F., Tomiyama, T., Sutherland, J.W., Kara, S. Herrmann, C., Duflou, J.R., 2012, Toward integrated product and process life cycle planning - An environmental perspective, Annals of the CIRP, 61/2: 681702.

[10] Sakao, T., Napolitano, N., Tronci, M., Sundin, E., Lindahl, M., 2008, How Are Product-Service Combined Offers Provided in Germany and Italy? - Analysis with Company Sizes and Countries -, Journal of Systems Science and Systems Engineering, 17/3: 367-381.

[11] Smith, D.J., 2013, Power-by-the-hour: the role of technology in reshaping business strategy at Rolls-Royce, Technology Analysis \& Strategic Management, 25/8: 987-1007.

[12] Settanni, E., Newnes, L.B., Thenent, N.E., Parry, G., Goh, Y.M., 2014, A through-life costing methodology for use in product-service-systems, International Journal of Production Economics, 153: 161-177.

[13] Marten, C., Gatzen, M.M., 2014, Decreasing operational cost of high performance oilfield services by lifecycle driven trade-offs in development, Annals of the CIRP, 63/1: 29-32.

[14] Arai, T. and Shimomura, Y., 2005, Service CAD System - Evaluation and Quantification, Annals of the CIRP, 54/1: 463-466. 